\title{
An audit of obesity data and concordance with diagnostic coding for patients admitted to Western Australian Country Health Service hospitals
}

\author{
AUTHORS \\ KIM McCLEAN MOccHlth\&Saf, ChOHSP ${ }^{1}$ \\ MARTYN CROSS PhD, MPH, BSc (Hons Tox), \\ ChOHSP, FAIHS, FAIOH, MACTRA ${ }^{2}$ \\ SUE REED PhD, MSc, MEngSc, BSc, $\mathrm{COH}, \mathrm{ClH}$, \\ ChOHSP, FAIOH, FAIHS ${ }^{2}$
}

\section{CORRESPONDING AUTHOR}

KIM McCLEAN Edith Cowan University, 270 Joondalup Drive, Joondalup, WA, Australia, 6027.

Email: k.mcclean@ecu.edu.au
1 Occupational Safety and Health Department, North Metropolitan Health Service, Western Australia, Australia.
2 School of Medical and Health Sciences, Edith Cowan University, Western Australia, Australia.

\section{ABSTRACT}

Objective: Accurate patient obesity data can be used to identify and mitigate patient manual handling risks to healthcare staff. This study investigates the accuracy of patient obesity data within the Western Australian Country Health Service (WACHS) and examines factors potentially affecting obesity data accuracy.

Background: Risk of injuries to healthcare staff are increasing due to rising patient obesity. Consistent increases in the prevalence of obesity in Australia have been recorded since 1995 and Australian obesity projections predict that $42 \%$ of the population will be obese in 2035 . To manage the increased risks of injuries to healthcare workers due to obese patient management, accurate healthcare data relating to patient obesity is required.

Design: Researchers examined records of patients admitted to WACHS hospitals with Type II Diabetes, which has confirmed links with obesity. Manual data extraction and comparison of obesity related data within patient medical records and electronic patient admission data was conducted to determine accuracy.
Results: Analysis of the patient data examination demonstrated poor recording of weight (67\%), height (24\%) and Body Mass Index (BMI) when weight and height measurements were recorded (10\%). Poor obesity data accuracy was also determined by low sensitivity results $(40 \%)$, high false negative results $(60 \%)$ and a Cohen's kappa value of 0.44 .

Discussion: The sensitivity result demonstrates that only $40 \%$ of obese patients were coded as obese when obesity is recorded in their medical files, and the false negative result demonstrates that where obesity notations were present in medical files, $60 \%$ of these cases were incorrectly coded as 'normal weighted'. There was only moderate agreement between the occurrences of coded obesity and the recorded obese patient notations in the medical files.

Conclusion: Further research is required to inform enhancements to improve obesity recording and coding accuracy, which will increase the collection of reliable obesity data that could be used to reduce obese patient handling risks to nurses and other healthcare staff. 
What is already known about the topic?

- Increasing Australian population obesity rates have been previously demonstrated, this increase corresponds with increasing numbers of obese patients being admitted into hospitals.

- Healthcare staff who care for obese patients are at increased risk of injuries when conducting patient handling tasks.

\section{What this paper adds:}

- A model to measure obesity accuracy utilising 14 data accuracy indicators was used, revealing poor obesity data accuracy and poor completeness of obesity data.
- Completeness of obesity data is influenced by time demands and workload of clinicians, breadth of clinical recording requirements, lack of organisational direction for the need of obesity data, and challenges in obtaining height measurements of patients who are mobility impaired, bed-ridden or unable to stand.

- Complete and accurate obesity data collections will result in increased ability to mitigate safety risks to healthcare staff who manage obese patients and may improve healthcare funding accuracy.

Keywords: Obesity, obese, patient handling, coding, administrative data.

\section{INTRODUCTION}

Increased risk of injuries to healthcare staff due to rising patient obesity is creating challenges for Australian healthcare organisations. Despite some Australian hospitals implementing 'no lift' policies, staff continue to experience musculoskeletal injuries due to obese (bariatric) patient handling requirements, particularly those staff who work in country or rural locations and the ageing healthcare workforce. Due to these injuries, which may affect both a worker's long term ability to perform work tasks and their home lifestyle, healthcare organisations are increasingly being affected by high insurance premiums and demands to improve staff safety.

The high prevalence of obesity within the Australian community is well documented, with the Australian Bureau of Statistics' (ABS) National Health Survey 2017-18 revealing that $31.3 \%$ of Australians aged 18 years and over were obese, ${ }^{1}$ and $35.6 \%$ were overweight. Consistent increases in the prevalence of obesity rates in Australia have been recorded since the ABS commenced collecting obesity data in 1995 , from $18.7 \%$ in $1995,24.4 \%$ in $2007-08$ to $31.3 \%$ in $2017-18 .{ }^{1-3}$ More disturbingly, Australian obesity projections predict that in $2035,42 \%$ of the population will be obese. ${ }^{4}$ This data demonstrates continuing risks for healthcare workers due to the population obesity prevalence increase and increases in obese patients requiring hospital admissions..$^{5-8}$

Accurate healthcare data relating to patient obesity is required to manage the increased risks of injuries to healthcare workers due to obese patient management. Healthcare organisations may have difficulties in designing and implementing evidence-based proactive risk management approaches due to lack of relevant data. Absence of obesity data may cause either ignorance of this risk or cause organisations to rely on anecdotal evidence of the risks. Seigal \& Ruoff promote the use of data to reduce organisational risks, assist organisational decision making ability and develop strategic direction and action plans to meet organisational needs. ${ }^{9}$ Similarly, Stanfill et al. assert that the need for data and data analysis in healthcare has never been bigger, ${ }^{10}$ and accurate coding and reporting of health diagnosis and conditions has become more crucial as healthcare data requirements have advanced.

In hospital environments, weight, BMI scores and/or notations of obesity are routinely captured for many healthcare requirements and are either manually or electronically recorded in patient files. Many Australian healthcare organisations are currently transitioning to the adoption of electronic health records, however a variety of methods of recording patient information is currently being used including the use of manual patient files, electronic records or hybrid models that involve manual files being scanned into patient admission databases. When patients are discharged from hospital, their admission records are analysed by clinical coding staff who assign up to 50 diagnosis codes to the patient electronic record. Obesity is coded when the condition is clinically observed and impacts the patient's management during their hospital admission by either requiring the commencement, alteration or adjustment of therapeutic treatment; requiring additional diagnostic procedures and/or requiring increased clinical care and/or monitoring. Diagnosis coding is undertaken according to the Australian Coding Standards $9^{\text {th }}$ Edition as defined by the ACCD. ${ }^{11}$ The Australian Coding Standards is a tool used by clinical coding staff that standardises code definitions and is used to ensure data consistency and integrity across all Australian health service providers.

Identifying obese patient admissions, and the related injury risks to healthcare workers who manage obese patients, is especially important for healthcare organisations in country or rural locations, as obesity rates are generally higher in country locations than metropolitan locations. ${ }^{14}$ In order to identify these risks in country hospital locations, an examination of obesity data accuracy is required. The 
Western Australian Country Health Service (WACHS) was selected for this study as it is the largest country (rural) health system in Australia, which provides an extensive range of health services across an area of 2.53 million square kilometres for an estimated population of 531,00o people.

Accuracy of obesity coding data has been examined in several international studies such as Martin et al. and Quan et al. which assessed variability between obesity coding and manual chart reviews, ${ }^{12,13}$ and both studies found large variances between the chart review results and clinical coding data. McClean, Cross and Reed conducted a pilot study into the accuracy of obese patient admission data recorded by the Western Australian Country Health Service in $2017,{ }^{15}$ which revealed poor recording of weight (59\%), height (15\%) and Body Mass Index (BMI) when weight and height measurements were recorded (8\%). Poor obesity data accuracy was also determined by low sensitivity results (41\%) and high false negative results (59\%). The sensitivity result demonstrated that, where obesity was recorded in patient files, only $41 \%$ were coded as obese. The obesity accuracy analysis methods used in the pilot study were successful and the study demonstrated a requirement for further analysis that may be able to inform enhancements to improve obesity recording and coding accuracy.

\section{AIM}

This research aims to determine if obese patient admission data recorded by WACHS provides sufficient accuracy to be used to implement risk mitigation strategies for nurses and other healthcare staff performing obese patient handling tasks.

\section{METHOD}

\section{DESIGN AND SETTING}

This study employed a retrospective audit of WACHS Patient Admission data and a manual examination of medical records at four WACHS regional hospitals (Sites A, B, C and $D)$. The hospitals were selected as they are larger health campuses in four different regions of Western Australia, and therefore are more likely to capture variations in rural obesity rates. The study examines the inclusion or absence of manual obesity notations, electronic obesity codes, weight, height and BMI recording. The application of BMI to measure obesity in this study was selected due to BMI being widely accepted as a reliable, inexpensive and efficient method of obesity measurement. ${ }^{16}$ In this study obesity is defined as a BMI equal to, or more than $30 \mathrm{~kg} / \mathrm{m},{ }^{2}$ which is in agreement with previous literature examining bariatric coding and ACCD definitions of obesity. ${ }^{5,11,13,18}$ The World Health Organization (WHO) defines the BMI calculation methodology as a person's weight in kilograms divided by the square of the person's height in metres $\left(\mathrm{kg} / \mathrm{m}^{2}\right){ }^{17}$
The study was approved by the Edith Cowan University (ECU) Human Research Ethics Committee, the WACHS Human Research Ethics Committee, the WACHS Research Governance Office and the WACHS Chief Executive.

\section{PATIENT CHARACTERISTICS}

De-identified patient admission data from the WebPAS ${ }^{\circledR}$, TOPAS $^{\circledR}$ and HCare ${ }^{\circledR}$ data administration systems was provided to the researchers by a WACHS Health Information Manager (HIM). The patient admission data inclusion criteria comprised of records for patients who were admitted to hospital for five days or more and discharged between 1 July 2015 and 30 June 2017, patients who were over the age of 18 at the time of hospital admission, and who had principal or additional diagnosis of "diabetes mellitus", which includes Type II diabetes. Diagnoses of Type II diabetes was selected as an inclusion criterion as it has a confirmed link with obesity. ${ }^{19,20}$ Records of patients who were admitted to hospital more than once in the audit period were included.

The data excluded records of patient boarders such as palliative care, and patients who use other health services such as outpatient treatments. Patients who had diagnosis terms of Type 1 Diabetes Mellitus, Family history of diabetes mellitus, Pre-existing diabetes mellitus, Type 1, or 'in pregnancy' were also excluded from the data. These exclusions were selected due to palliative care and outpatient services not conforming to the research focus of examining patient admission to hospitals, lack of confirmed links between obesity and Type 1 diabetes, and pregnancy-related diabetes being a potentially temporary condition.

\section{PROCEDURE}

The examination of obesity coding was conducted by identification of obesity codes within the selected patient records that met the inclusion criteria. The principal and additional diagnosis codes relating to obesity as defined by the Australian Coding Standards $9^{\text {th }}$ Edition are: ${ }^{11}$

- E66-Obesity;

- E66.o - Obesity due to excess calories;

- E66.1 - Drug induced obesity;

- E66.2 - Extreme obesity with alveolar hypoventilation;

- E66.8 - Other obesity;

- E66.9-Obesity, unspecified; and

- U78.1 - Endocrine, nutritional and metabolic diseases obesity

WACHS Health Information Managers determined patients and their corresponding episodes (admission and discharge dates) that fell within the study inclusion criteria. A manual examination of the medical files was then conducted to examine the inclusion or absence of obesity recording, and weight, height and BMI recording. Sections of the medical files examined included but were not limited to Emergency 
Department notes, nursing admission screening tools, handover/interim care plans, progress notes, medication charts, anaesthetic records, insulin charts, malnutrition screening tools, dietetics assessments, fluid balance charts, perioperative pathway forms and discharge summaries. The principal researcher undertook training on medical file examination techniques before the manual file examination to ensure sound data extraction methods were met.

\section{DATA ANALYSIS}

A comparative assessment was conducted against the medical file data and the occurrence of principal and additional diagnosis codes relating to obesity, being the E66 coding suite and $U_{78.1}$ additional diagnosis code. Seven quantitative techniques were utilised to examine the accuracy of the patient admission data compared with medical file reviews: sensitivity, specificity, positive predictive value (PPV), negative predictive value (NPV), false negative rates, false positive rates and Cohen's Kappa values. This analysis methodology is commonly used in clinical examinations of interventions and comparisons, and is supported by several clinical research projects, including Lee et al. and Ho et al. ${ }^{21,22}$ Seven additional methods of quantitative analysis were also applied: percentage coded as obese, weight recorded, height recorded, BMI calculated, height and weight recorded with no BMI, obesity or BMI notations recorded and obesity or BMI notations recorded but height and weight not recorded.

Measuring sensitivity determined the degree of obesity recording in the patient admission data when it was first present in the medical files, while specificity measured the absence of obesity conditions in the patient admission data if the condition is absent in the medical files. Accuracy of the clinical coding of obesity-related conditions was examined by the analysis of PPVs and NPVs. Negative predictive value firstly examines the absence of obesity coding and then examines the absence of obesity notations in medical files, conversely positive predictive value firstly examines the cases that were coded as obese and then examines the occurrences of obesity notations in medical files. Cohen's Kappa values determined the agreement between the patient admission data and the medical file data.

\section{RESULTS}

The summary of the results of the statistical analysis of obesity data accuracy indicators is shown in Table 1.

The study included 590 records consisting of those of 297 males (50.3\%) and 293 females (49.7\%) aged between 18 and 98 years. Obesity was coded in $10.8 \%$ of all patients, with weight being recorded in $67.3 \%$ of all patients and height being recorded in $24.1 \%$ of patients. BMI was calculated in $10.8 \%$ of all patients, and of the patients who had height and weight recorded, $62 \%$ of patients did not have BMI recorded. Obesity or BMI notations were recorded in $19.4 \%$ of all patients, however 9.3\% of obesity or BMI notations were not supported by height or weight records.

Analysis of average sensitivity and specificity between obesity coding and obesity recordings in medical files resulted in 40 and $96.2 \%$ respectively. Analysis of average negative predictive values and positive predictive value resulted in 86.9 and $71.8 \%$ respectively. The average false positive outcome was $3.8 \%$, while the average false negative outcome

TABLE 1: WACHS PATIENT ADMISSION OBESITY ACCURACY AND INTER-RATER RELIABILITY ANALYSIS

\begin{tabular}{|c|c|c|c|c|c|c|c|}
\hline & All & Male & Female & Site A & Site B & Site C & Site D \\
\hline Records within research criteria & 847 & 422 & 425 & 209 & 199 & 219 & 220 \\
\hline Records audited & 590 & 297 & 293 & 166 & 100 & 158 & 166 \\
\hline Coded as obese $(n, \%)$ & $64(10.8 \%)$ & $31(10.4 \%)$ & $33(11.3 \%)$ & $14(8.4 \%)$ & $3(3.0 \%)$ & $16(10.1 \%)$ & $31(18.67 \%)$ \\
\hline Weight recorded & $397(67.3 \%)$ & $190(64.0 \%)$ & 207 (70.6\%) & $94(56.6 \%)$ & $70(70.0 \%)$ & $100(63.3 \%)$ & $133(80.1 \%)$ \\
\hline Height recorded & $142(24.1 \%)$ & $63(21.2 \%)$ & $79(26.6 \%)$ & $21(12.6 \%)$ & 9 (9.0\%) & $33(20.1 \%)$ & $79(47.6 \%)$ \\
\hline BMI calculated & $64(10.8 \%)$ & $20(6.7 \%)$ & $44(15.0 \%$ & $10(6.0 \%)$ & $7(7.0 \%)$ & $19(12.0 \%)$ & $28(16.9 \%)$ \\
\hline Height and weight recorded, no BMI & $88(62.0 \%)$ & $44(69.8 \%)$ & $44(55.7 \%)$ & $12(57.1 \%)$ & $8(88.8 \%)$ & $14(42.4 \%)$ & $54(68.3 \%)$ \\
\hline Obesity or BMI notations recorded & $115(19.4 \%)$ & $47(15.8 \%)$ & $68(23.2 \%)$ & $27(16.2 \%)$ & $12(12.0 \%)$ & $34(21.5 \%)$ & $42(25.3 \%)$ \\
\hline $\begin{array}{l}\text { Obesity or BMI notations recorded but } \\
\text { height and weight not recorded }\end{array}$ & 55 (9.3\%) & $23(7.7 \%)$ & $32(10.9 \%)$ & $21(12.6 \%)$ & $1111.0 \%)$ & $12(7.5 \%)$ & $11(6.6 \%)$ \\
\hline Sensitivity & $40.0 \%$ & $42.6 \%$ & $38.2 \%$ & $48.1 \%$ & $8.3 \%$ & $35.3 \%$ & $47.6 \%$ \\
\hline Specificity & $96.2 \%$ & $95.6 \%$ & $96.9 \%$ & $99.3 \%$ & $97.7 \%$ & $96.8 \%$ & $91.1 \%$ \\
\hline NPV & $86.9 \%$ & $89.9 \%$ & $83.9 \%$ & $90.8 \%$ & $88.6 \%$ & $84.5 \%$ & $83.7 \%$ \\
\hline PPV & $71.8 \%$ & $64.5 \%$ & $78.8 \%$ & $92.9 \%$ & $33.3 \%$ & $75.0 \%$ & $64.5 \%$ \\
\hline False positive & $3.8 \%$ & $4.4 \%$ & $3.1 \%$ & $0.7 \%$ & $2.3 \%$ & $3.2 \%$ & $8.9 \%$ \\
\hline False negative & $60.0 \%$ & $57.4 \%$ & $61.8 \%$ & $51.9 \%$ & $91.7 \%$ & $64.7 \%$ & $52.4 \%$ \\
\hline Kappa & 0.44 & 0.44 & 0.43 & 0.59 & 0.09 & 0.40 & 0.42 \\
\hline
\end{tabular}




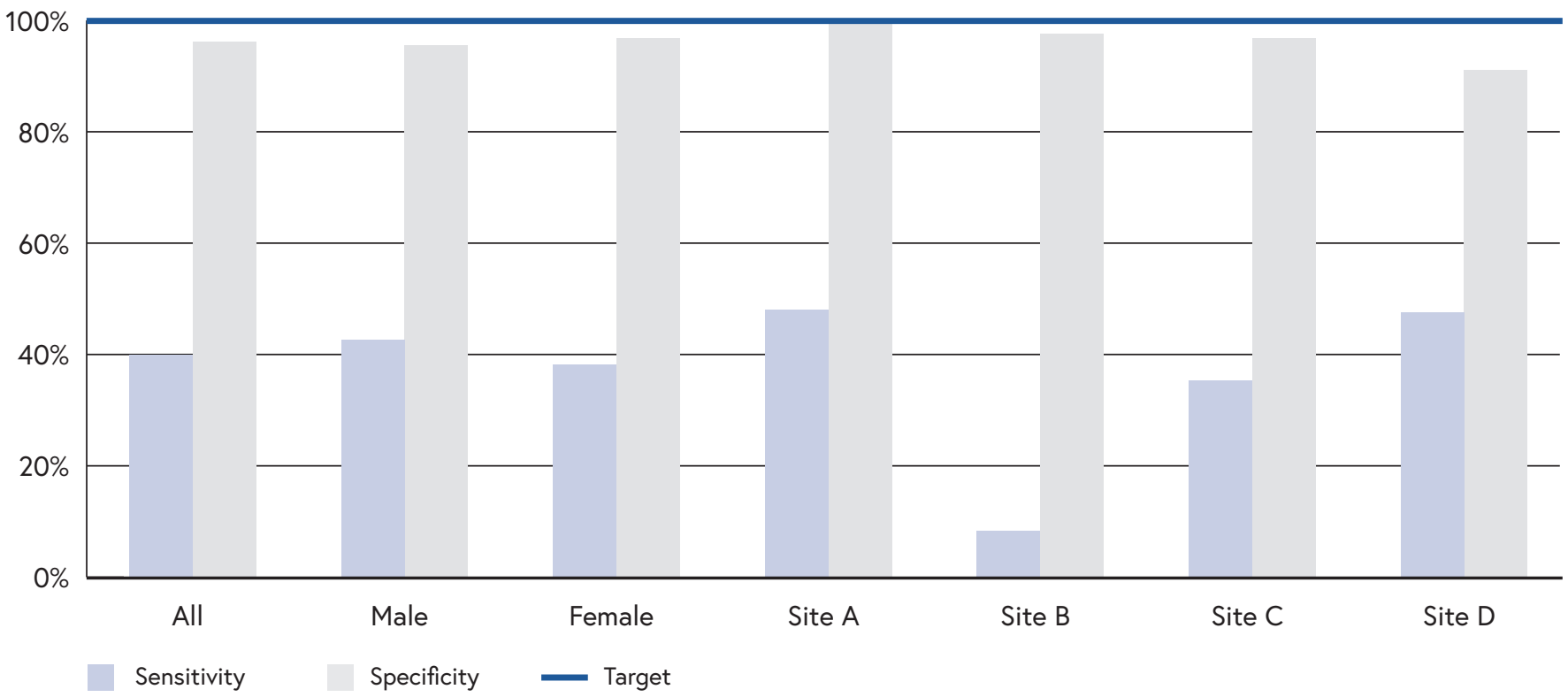

GRAPH 1: WACHS OBESITY DATA SENSITIVITY AND SPECIFICITY

was 60\%. The average Cohen's kappa value was o.44. Graph 1 displays the sensitivity and specificity results, and includes an aspirational specificity and sensitivity targets established by the study authors of $100 \%$ in order to support enhanced obesity coding accuracy.

As a result of patient records being archived in offsite locations due to limited hospital storage, patient records being used on hospital wards or in outpatient departments due to ongoing treatment, patients records being utilised for clinical coding or limitations of researcher availability (as discussed in limitations section), 257 records were unable to be examined.

\section{DISCUSSION}

Generally, poor obesity accuracy of the patient admission data was demonstrated in the comparative data analysis of obese patient coding and obesity recordings in medical files. Low average sensitivity results (40\%), high average false negative results (6o\%) and the Cohen's kappa value of 0.44 all support findings of poor accuracy of the obese patient admission data. The sensitivity result demonstrates that, where obesity was recorded in patient files, only $40 \%$ were coded as obese, similarly the average false negative result of $60 \%$ demonstrated that of all cases that should have been coded as obese due to the inclusion of obesity calculations or notations in the medical files, $60 \%$ of these cases were incorrectly coded as 'normal weighted'. Cohen's kappa value demonstrates correlation between occurrences of coded obesity and the recorded obese patient notations in the medical files, with the closer the result value is to one, the higher the correlation. The study's average Cohen's kappa value result of 0.44 demonstrated only moderate agreement. The positive predictive value of $71.8 \%$ did however demonstrate moderate levels of accuracy when clinical coding staff are coding obesity and there is evidence of obesity in the clinical file records.

Conversely, high accuracy of coding non-obese patients was demonstrated by the high average specificity result (96.2\%), and high average negative predictive values (86.9\%). The specificity result demonstrated that where there are no obesity notations recorded, clinical coders are correctly coding these patients as normal weighted in $96.2 \%$ of all occurrences. Similarly, the average negative predictive value result of $86.9 \%$ demonstrated that of all 'normal weighted' coded patients, $86.9 \%$ of these patients did not have obesity notations recorded in medical files.

There was poor completeness of weight, height and BMI measurement data in patient files (67.3, 24.1 and 10.8\% respectively). While scales to measure patient weight are commonly available in healthcare organisations, equipment to measure patient height is often lacking which could contribute to the low recording of patient height, which negatively impacts clinician ability to conduct BMI calculations. Wall mounted and calibrated height measurement tools should be readily available in hospital wards to measure patient height. For patients who are mobility impaired, bed-ridden or unable to stand due to their health conditions, healthcare workers may be challenged to obtain height data, an essential measurement used in BMI calculations that translate to obesity coding. Several evidence-based methods of obtaining reliable height measurements from bone measurements are available, such as the Ulna length method, Demi-span method or knee height method. These methods provide accurate estimates of stature in normally proportioned adults, and clinicians should be trained in the use of these alternate height measurement techniques. ${ }^{23-25}$ 
Poor completeness of weight, height and BMI data may also be due to time demands and workload of clinicians, breadth of total clinical recording requirements, and lack of organisational direction for the need of this data. Galinski, Hudock \& Streit highlight that approximately $75 \%$ of morbidly obese patients have at least one co-morbid condition. ${ }^{5}$ The immediate focus of both doctors and nurses is the treatment of the condition causing the patient to be admitted. This concentrated focus on the health condition(s) causing hospital admission is also mirrored in coding practices, where primary health conditions are coded in the first instance, as they are important indicators for health condition data and funding provision by the Western Australian government.

Stanfill et al. asserts that data recording and analysis in healthcare has increased substantially over time, ${ }^{10}$ and high data collection requirements that healthcare organisations place on their clinicians may affect their prioritisation of obesity data recording. In addition to the competing data priorities, lack of clinician awareness of the importance and use of obesity data for clinical, safety and funding purposes are likely contributors to the absence of obesity data and notations in files of obese patients. Insufficient organisational prioritisation of obesity data recording and use of the data itself, and related lack of auditing of the obesity data recording, may also be a factor that further influences low obesity data recording. As healthcare organisations progress to electronic patient records, consideration should be given to mandatory recording fields for patient height and weight, automated BMI calculations, and indicators or 'file flags' for patients who are obese and require additional patient care measures.

The requirement for obesity codes to be added to the coding data set in an 'opt in' data approach is likely to be an additional contributing factor that will affect obesity coding accuracy. If obesity is not coded, the default data position indicates 'normal weighted'. Another challenge to obesity data accuracy is requirements within the Australian Coding Standards $9^{\text {th }}$ Edition for coders to only code patients as obese if a BMI score is provided or a clinical notation detailing patient obesity is explicitly recorded. Currently, if weight and height are available within the medical record, coders are not able to calculate and code BMI. In practice, however, weight, height and BMI recording itself is low. Even when weight and height is recorded in the patient's medical file, in $62 \%$ of these instances the measurements are not translated to a BMI calculation by the clinician.

Furthermore, clinical notes which indicate a high BMI has been observed (such as $\uparrow$ BMI) are not deemed to be sufficient detail to be coded. While the data challenges do not impact the clinical treatment of obese patients, they do affect an organisation's ability to proactively manage obese patient handling risks by the identification of current risks and predicting the extent of future risks. The ability for clinical coders to use height, weight and BMI scores to code obesity, and the resulting impact on obesity data accuracy should be explored further. Again, with the adoption of electronic patient records, mandatory recording of height and weight, automated BMI calculations, and a check box field that indicates an impact to clinical care may be worthy of further examination.

It appears that a degree of obesity notations by clinical staff are likely due to visible observations of obesity. Within the examined medical files, there were 115 instances of BMI or obesity notations recorded in the clinical notes, of which 55 records were not supported by a recorded patient weight or height. Of the 115 records with obesity notations, only 64 medical files contained BMI scores. Furthermore, visual obesity identification may be affected by obesity normalisation due to increased prevalence of both obese patient presentations within the healthcare setting and in the community. As a result of continually rising prevalence of obesity in Australia, society's acceptance of heavier body weights as 'normal' is also increasing, such as described by Maynard and others. ${ }^{26}$ Therefore, a clinician's visual assessment may underestimate BMI. While clinical notations of obesity are important, these are likely to be subjective observations and should be supported by measured weight, height and BMI data.

Finally, lack of obesity records and coded data has healthcare funding implications. All diagnosis codes are processed through a series of calculations including Diagnosis-related Groups (DRGs) and National Weighted Activity Units (NWAUs) that result in hospital funding for the patient service that is determined by the Activity Based Funding $(\mathrm{ABF})$ system. If treatment is provided for obese patients where patient care is affected by the obesity condition, lack of obesity recording or coding will result in these factors not being included in the funding calculations. For example, this will mean that instances of change in patient care such as increased staffing requirements for lifting, turning or toileting of obese patients, use of bariatric equipment, increases in anaesthetic or medication doses, change in rehabilitation approaches, and change in clinical risk categories for obese maternity patients will not be included in the funding calculations. The impact on healthcare funding due to lack of obesity coding or coding inaccuracy should also be explored further.

\section{LIMITATIONS}

Examining the clinical methods of obtaining the data contained within patient files and the accuracy of this data is outside of the scope of the research study. The data within patient files is recorded by trained clinical staff and is considered to be the gold standard for analysis and comparison. Due to the distance between WACHS hospitals and associated travel requirements for researchers to attend 
rural hospital locations to conduct manual file examinations, it is acknowledged that a limitation of this study is researcher availability. While the data collection provided valid results, increased data collections may be required to inform future research which will require increased researcher availability. The ongoing adoption of electronic health records by healthcare organisations will likely allow researchers to manually review patient files at central locations, which may reduce this limitation in the future. An additional limitation due to researcher availability was the inclusion of patients only with Type II Diabetes. As diabetes is strongly linked to obesity, rates of obesity coding in the patient administrative data may be higher than in the general population. Expanding the patient inclusion criteria will allow an examination of obesity recording accuracy of the general patient population.

\section{CONCLUSION}

Healthcare organisations have a legal obligation under Occupational Safety and Health/Work Health and Safety legislation to ensure safe workplaces are provided to employees who manage obese patients. Anecdotal awareness of increased obese patient admissions and corresponding increased risks of injuries to healthcare staff are acknowledged by healthcare executives, nursing staff and Occupational Health and Safety staff, however the ability for healthcare organisations to identify obese patient admission trends and adopt risk management approaches is impacted by low obese patient admission coding accuracy, particularly in the areas of sensitivity and false negatives.

\section{IMPLICATIONS FOR RESEARCH, POLICY AND PRACTICE}

Enhanced methods to record BMI and obesity should be considered, including mandatory recording of weight and height and progressing organisational movement to electronic healthcare records. Automated BMI calculations and user-friendly methods to indicate patient care impacts of obesity will assist clinicians to easily record obesity data and simplify obesity coding, which will increase obesity data accuracy. Until full adoption of electronic health records, healthcare organisations should promote the importance of obesity data and increase clinical staff awareness of the requirement for improved height, weight and BMI recording, and the potential use of this data for non-clinical uses such as obese patient handling risk mitigation. The impact of current obesity coding processes should be examined, particularly the absence of obesity recording resulting in the default data coding position indicating 'normal weighted'. Furthermore, investigation of alternative coding methods to obtain obesity recordings should be conducted such as allowing clinical coders the ability to determine BMI categories if height and weight measurements are available in medical files.

\section{CONFLICT OF INTEREST}

The authors of this paper declare no conflict of interest.

\section{ACKNOWLEDGEMENTS}

The authors thank the Western Australian Country Health Service (WACHS) for their contributions to this study, South West Regional Director Kerry Windsor and the hospitals and staff who assisted with the study particularly Meegan Kidd, Program Officer Clinical Coding and regional Clinical Coding staff.

\section{FUNDING SUPPORT}

This study was supported in-kind by WACHS.

\section{REFERENCES}

1 Australian Bureau of Statistics (ABS). National health survey: first results, 2017-18 (cat. no. 4364.0.55.001). Australian Bureau of Statistics. Canberra. 2019. [cited 2019 Nov 16] Available from: https://www.abs.gov.au/statistics/health/health-conditionsand-risks/national-health-survey-first-results/latest-release

2 Australian Bureau of Statistics (ABS). National health survey: first results, 1995 (cat. no. 4392.0). Australian Bureau of Statistics. Canberra. 1996. [cited 2019 Nov 16]. Available from: https://www.abs.gov.au/AUSSTATS/abs@.nsf/ Lookup/4392.0Main+Features11995?OpenDocument

3 Australian Bureau of Statistics (ABS). Overweight and obesity in adults in Australia: a snapshot, 2007-08 (cat. no. 4842.0.55.001). Canberra. 2011. [cited 2019 Nov 18] Available from: https://www.abs.gov.au/ausstats/abs@.nsf/ $\mathrm{mf} / 4842.0 .55 .001 /$

4 Walls HL, Magliano DJ, Stevenson CE, Backholer K, Mannan $H R$, Shaw JE, et al. Projected progression of the prevalence of obesity in Australia. Obesity. 2012; 20(4): 872-78. Available from: https://doi.org/10.1038/oby.2010.338

5 Galinsky T, Hudock S, Streit J. Addressing the need for research on bariatric patient handling. Rehabil Nurs . 2010; 35(6): $242-$ 47. Available from: https://doi.org/10.1002/j.2048-7940.2010. tb00054.x

6 Muir M, Archer-Heese G, McLean D, Bodnar S, Rock B. Handling of the bariatric patient in critical care: a case study of lessons learned. Crit Care Nurs Clin North Am. 2007; 19(2): 223-40. Available from: https://doi.org/10.1016/j.ccell.2007.02.010

7 Australian National Preventative Health Agency (ANPHA). Obesity: Prevalence trends in Australia. Sydney. 2014 [cited 2016 Nov 14]; Available from: http://sydney.edu.au/medicine/ research/units/boden/ANPHA\%200besity\%20Prevalence\%20 Trends.pdf

8 Pieracci F, Barie P, Pomp A. Critical care of the bariatric patient Crit Care Med. 2006; 34(6): 1796-1804. Available from: https:// doi.org/10.1097/01.CCM.0000217216.84983.0A

9 Seigal D, Ruoff G. Data as a catalyst for change: Stories from the frontlines. J Healthc Risk Manag. 2015; 34(3):18-25. Available from: https://doi-org.ezproxy.ecu.edu.au/10.1002/ jhrm.21161 
10 Stanfill MH, Williams M, Fenton SH, Jenders RA, Hersh WR. A systematic literature review of automated clinical coding and classification systems. J Am Med Inform Assoc. 2010; 17(6): 646-51.

11 Australian Consortium for Classification Development. Australian Coding Standard 9th Edition. New South Wales. 2015. Available on request by ACCD https://www.accd.net.au

12 Martin B, Chen G, Graham M, Quan H. Coding of obesity in administrative hospital discharge abstract data: accuracy and impact for future research studies. BMC Health Serv Res. 2014; 14(70): 1-8. Available from: https://link.springer.com/ article/10.1186/1472-6963-14-70

13 Quan H, Li B, Saunders D, Parsons GA, Nilsson Cl, Alibhai A, et al. Assessing validity of ICD-9-CM and ICD-10 administrative data in recording clinical conditions in a unique dually coded database. Health Serv Res. 2008; 43(4): 1424-41. Available from: https://doi-org.ezproxy.ecu.edu.au/10.1111/j.14756773.2007.00822.x

14 Australian Bureau of Statistics (ABS). Overweight and obesity in adults, Australia, 2004-05 (cat. no. 4719.0). Canberra. 2008. [cited 2017 Oct 8]. Available from http://www.abs.gov.au/ ausstats/abs@.nsf/mf/4719.0/.

15 McClean K, Cross M, Reed S. Accuracy of obese patient admission data recorded by the Western Australian Country Health Service: A pilot study. J Health Saf Environ. 2019; 35(1):107-17.

16 Lemay CA, Cashman SB, Savageau JA, Reidy PA. Effect of a low-cost intervention on recording body mass index in patients' records. J Nurs Scholarsh. 2004; 36(4): 312-15. Available from https://doi-org.ezproxy.ecu.edu.au/10.1111/j.1547_ 5069.2004.04057.x

17 World Health Organization. Preventing and managing the global epidemic: WHO obesity technical report series 894. Geneva. 2000. [cited 2018 May 1]. Available from http://www.who.int/ iris/handle/10665/42330.

18 Grant P, Lipscomb D. Diagnosis of obesity and clinical implications. Pract Diabetes Int. 2008; 25(9): 376. Available from https://doi-org.ezproxy.ecu.edu.au/10.1002/pdi.1313

19 American Society for Metabolic and Bariatric Surgery. Fact sheet: Obesity in America. American Society for Metabolic and Bariatric Surgery. Florida. 2013. [cited 2016 Mar 16]. Available from https://asmbs.org/app/uploads/2014/06/Obesity-inAmerica-1.pdf

20 Medical Research Council United Kingdom. Diabetes: facts and stats. Diabetes UK. London. 2015. [cited 2016 Mar 21] Available from https://www.mrc.ac.uk/documents/pdf/diabetes-ukfacts-and-stats-june-2015

21 Lee YYC, Roberts CL, Young J, Dobbins T. Using hospital discharge data to identify incident pregnancy-associated cancers: a validation study. BMC Pregnancy Childbirth. 2013; 13(37): 1-6. Available from https://link.springer.com/ article/10.1186/1471-2393-13-37

22 Ho ML, Lawrence N, Van Walraven C, Manuel D, Keely E, Malcolm J, et al. The accuracy of using integrated electronic health care data to identify patients with undiagnosed diabetes mellitus. J Eval Clin Pract. 2012; 18(3): 606-11. Available from https://doi.org/10.1111/j.1365-2753.2011.01633.x

23 Han TS, Lean ME. Lower leg length as an index of stature in adults. Int J Obes Relat Metab Disord. 1996; 20(1): 21-7. Available from https://pubmed.ncbi.nlm.nih.gov/8788318/
24 Hickson M, Frost G. A comparison of three methods for estimating height in the acutely ill elderly population. J Hum Nutr Diet. 2003; 16(1): 13-20. Available from: https://doi.org/10.1046/j.1365-277x.2003.00416.x

25 Jarzem PF, Gledhill RB. Predicting height from arm measurements. J Pediatr Orthop. 1993; 13(6): 761-65. Available from: https://doi.org/10.1097/01241398-199311000-00014

26 Maynard LM, Serdula MK, Galuska DA, Gillespie C, Mokdad, AH. Secular trends in desired weight of adults. Int J Obes. 2006; 30(9):1375-81. Available from https://search-proquest-com. ezproxy.ecu.edu.au/docview/219271488 